\title{
Effects of Melatonin Treatment in Septic Newborns
}

\author{
ELOISA GITTO, MALGORZATA KARBOWNIK, RUSSEL J. REITER, DUN XIAN TAN, \\ SALVATORE CUZZOCREA, PIETRO CHIURAZZI, SANTA CORDARO, GIUSEPPINA CORONA, \\ GIUSEPPE TRIMARCHI, AND IGNAZIO BARBERI \\ Department of Cellular and Structural Biology, The University of Texas Health Science Center at San \\ Antonio, San Antonio, TX 78229-3900, U.S.A. [E.G., M.K., R.J.R., D.X.T.]; Department of Pediatrics, \\ Neonatal Intensive Care Unit, University of Messina, Italy [E.G., P.C., S.Cordaro, G.C., I.B.]; \\ Department of Thyroidology, Institute of Endocrinology, Medical University of Łodz, Poland [M.K.]; \\ Institute of Pharmacology, University of Messina, Italy [S.Cuzzocrea.]; Department of Statistics, University of \\ Messina, Italy [G.T.]
}

\begin{abstract}
ABST
Free radicals have been implicated in the pathogenesis of
neonatal sepsis and its complications. This study was conducted
to determine the changes in the clinical status and the serum
levels of lipid peroxidation products [malondialdehyde (MDA)
and 4-hydroxylalkenals (4-HDA)] in 10 septic newborns treated
with the antioxidant melatonin given within the first 12 h after
diagnosis. Ten other septic newborns in a comparable state were
used as "septic" controls, while 10 healthy newborns served as
normal controls. A total of 20 mg melatonin was administered
orally in two doses of 10 mg each, with a 1-h interval. One blood
sample was collected before melatonin administration and two
additional blood samples (at 1 and 4 h) were collected after
melatonin administration to assess serum levels of lipid peroxi-
dation products. Serum MDA +4 -HDA concentrations in new-
borns with sepsis were significantly higher than those in healthy
infants without sepsis; in contrast, in septic newborns treated
with melatonin there was a significant reduction ( $p<0.05)$ of
MDA +4 -HDA to the levels in the normal controls at both 1 and
$4 \mathrm{~h}(p<0.05)$. Melatonin also improved the clinical outcome of
the septic newborns as judged by measurement of sepsis-related
\end{abstract}
serum parameters after 24 and $48 \mathrm{~h}$. Three of 10 septic children who were not treated with melatonin died within $72 \mathrm{~h}$ after diagnosis of sepsis; none of the 10 septic newborns treated with melatonin died. To our knowledge, this is the first study where melatonin was given to human newborns. (Pediatr Res 50: 756-760, 2001)
MDA, malondialdehyde
Abbreviations
4, HDA, 4-hydroxylalkanals
ROS, reactive oxygen species
HPS, highly probable sepsis
PRS, probable sepsis
POS, possible sepsis
NS, no sepsis
WBC, white blood cell count
ANC, absolute neutrophil count
PLT, platelets
CRP, $C$ reactive protein
TNF, alpha, tumor necrosis factor alpha

Sepsis represents a serious problem in newborns with an incidence of 1 to 10 cases per 1000 live births, with even higher rates in low-birth-weight neonates. Hospital-acquired infections in neonatal intensive care units may also occur as frequently as 30 infections per 100 patients. Mortality rates in septic newborns are $30 \%$ to $50 \%$ (1). Sepsis is characterized by alterations in body temperature, hypotension, hypoperfusion with cellular injury, and organ failure, often resulting in death (2). There are several reports that suggest that reactive oxygen

Received October 13, 2000; accepted February 6, 2001.

Corresponding author: Russel J. Reiter, Department of Cellular and Structural Biology, The University of Texas Health Science Center at San Antonio, Mail Code 7762, 7703 Floyd Curl Drive, San Antonio, Texas 78229-3900, U.S.A.; e-mail: reiter@uthscsa.edu E. Gitto was supported by a fellowship of the "Miglierina-Griffini" Foundation. species (ROS) play a significant role in the pathogenesis of neonatal sepsis and its complications $(3,4)$.

While it has been suggested that antioxidants be used to counteract the toxicity of oxygen radicals and ROS (5), few trials have actually been performed. Oxygen toxicity can be ameliorated either by reducing ROS generation or by neutralizing these reactive species once they are formed. Newborns have less protection against oxidation (6). In comparison with healthy adults, lower levels of plasma antioxidants such as vitamin E, $\beta$-carotene, and sulfhydryl groups, lower levels of plasma metal binding proteins such as ceruloplasmin and transferrin, and reduced activity of erythrocyte superoxide dismutase are typical of newborn infants. Furthermore, infants frequently have higher plasma levels of 
nontransferrin-bound iron and higher erythrocyte free iron than adults.

Melatonin, an endogenously produced indolamine formed in adult humans, but only minimally so in neonates, is a highly effective antioxidant and free radical scavenger $(7,8)$. This study assessed the efficacy of antioxidant therapy with melatonin in neonates with sepsis. Serum levels of lipid peroxidation products malondialdehyde [malondialdehyde (MDA) and 4-hydroxylalkanals (4-HDA)] were examined before and after 1 and $4 \mathrm{~h}$ of melatonin treatment. Clinical status and sepsisrelated serum parameters were also evaluated at 24 and $48 \mathrm{~h}$ after melatonin administration.

\section{METHODS}

Patients and control samples. This study was performed in the Neonatal Intensive Care Unit of the University of Messina and was approved by the Ethical Committee of the University Hospital and performed only after parental consent. Twenty newborns with sepsis diagnosed within the previous $12 \mathrm{~h}$ were investigated along with 10 healthy infants. All septic patients were diagnosed as having highly probable sepsis (HPS) or probable sepsis (PRS) according to the criteria reported in Table 1 and were treated with antibiotics according to standard protocols.

Gestational age, birth weight, and Apgar index at 5 min for all newborns included in the three groups (healthy newborns, untreated septic newborns, septic newborns treated with melatonin) are listed in Table 2.

Table 1. Criteria employed for defining the sepsis score

High probable sepsis (HPS) At least 3 sepsis-related clinical signs* $\mathrm{CRP}>5 \mathrm{mg} / \mathrm{ml}$

At least 2 other altered serum parameters $\dagger$ Blood culture: positive or negative

Probable sepsis (PRS) Less than 3 sepsis-related clinical signs* $\mathrm{CRP}>5 \mathrm{mg} / \mathrm{ml}$

At least 2 other altered serum parameters $\dagger$ Blood culture: negative

Possible sepsis (POS) Less than 3 sepsis-related clinical signs* $\mathrm{CRP}<5 \mathrm{mg} / \mathrm{ml}$

Less than 2 other altered serum parameters $\dagger$ Blood culture: negative

No sepsis (NS)

No sepsis-related clinical signs*

$\mathrm{CRP}<5 \mathrm{mg} / \mathrm{ml}$

No altered serum parameters $\dagger$

Blood culture: negative

* Sepsis-related clinical signs: temperature instability; apneic spells; need for supplemented oxygen; need for ventilation; tachycardia/bradycardia; hypotension; feeding intolerance; abdominal distension; necrotizing enterocolitis.

$\dagger$ Serum parameters other than CRP: white blood cells (WBC) count; absolute neutrophil count (ANC); platelet (PLT) count.
Blood collection and treatment. Blood was collected from the umbilical cord or a peripheral vein of all septic and healthy infants before any treatment. In a random manner, 10 septic infants received orally a total of $20 \mathrm{mg}$ melatonin (2 doses of $10 \mathrm{mg}$ separated by a 1-h interval) dissolved in $5 \mathrm{~mL}$ of a 1:90 mixture of ethanol/physiologic saline. Melatonin was chromatographically pure (Helsinn Chemical Co., Biasca, Switzerland). The second group of 10 septic newborns did not receive melatonin. One hour and $4 \mathrm{~h}$ after melatonin administration, second and third blood samples were collected from all septic infants. Serum was separated by centrifugation at $1500 \times \mathrm{g}$ for $10 \mathrm{~min}$ and was stored at $-20^{\circ} \mathrm{C}$ until MDA + 4-HDA analysis. Blood samples were also obtained from all newborns (healthy, septic untreated, and septic treated with melatonin) at 24 and $48 \mathrm{~h}$ after melatonin administration to assess serum parameters listed in Table 1.

Measurement of lipid peroxidation. The levels of MDA + 4-HDA in each serum sample were measured using a commercial kit (586 test kit, Calbiochem, La Jolla, CA, U.S.A.). This assay is based on the reaction of a chromogenic reagent with MDA and 4-HDA to yield a stable chromophore, with maximal absorbance at $586 \mathrm{~nm}$. The tubes were mixed, closed and incubated for $40 \mathrm{~min}$ in a water bath at $45^{\circ} \mathrm{C}$. Samples were then cooled on ice and their absorbance were measured with a UltraSpec-3000 spectrophotometer.

Statistical analysis. The data in Tables 2, $3 \mathrm{a}$ and $3 \mathrm{~b}$ are presented as mean values \pm standard deviations. Nonparametric statistical tests were used to evaluate the significance of the differences between data sets in Tables $3 \mathrm{c}$ and $3 \mathrm{~d}$. In particular, Friedman's test was used when comparing values at different time points in the treated and untreated septic groups (Table 3c) and U-test of Mann-Whitney was used to compare the differences between treated and untreated newborns at the same time points (Table 3d). The data in Figure 1 were analyzed using a one-way analysis of variance (ANOVA) followed by the Student-Newman-Keuls $t$ test.

\section{RESULTS}

The three groups of newborns (10 healthy, 10 untreated septic and 10 septic neonates treated with melatonin) did not differ from each other with respect to gestational age or birth weight (Table 2). Obviously, the mean Apgar index at $5 \mathrm{~min}$ in healthy newborns $(9.70 \pm 0.48)$ was higher than that of both treated and untreated septic newborns $(7.50 \pm 1.08)$. Also there were no differences between the treated and untreated septic infants with respect to mode of delivery, duration of ruptured membranes, evidence of maternal fever, or antenatal steroid use.

Tables $3 \mathrm{a}$ and $3 \mathrm{~b}$ show the changes in serum parameters over three time points in the 10 melatonin-treated and 10 untreated

Table 2. Gestational age, birth weight, and Apgar index in the three groups of newborns

\begin{tabular}{|c|c|c|c|c|c|c|}
\hline & \multicolumn{2}{|c|}{ Healthy newborns } & \multicolumn{2}{|c|}{ Untreated septic } & \multicolumn{2}{|c|}{ Treated septic } \\
\hline & Mean & SD & Mean & SD & Mean & SD \\
\hline Gestational age (wk) & 39.30 & 1.06 & 38.60 & 1.17 & 39.10 & 1.29 \\
\hline Apgar index & 9.70 & 0.48 & 7.50 & 1.08 & 7.50 & 0.97 \\
\hline
\end{tabular}


Table 3. Comparison of serum parameters between treated and untreated septic infants at different time points

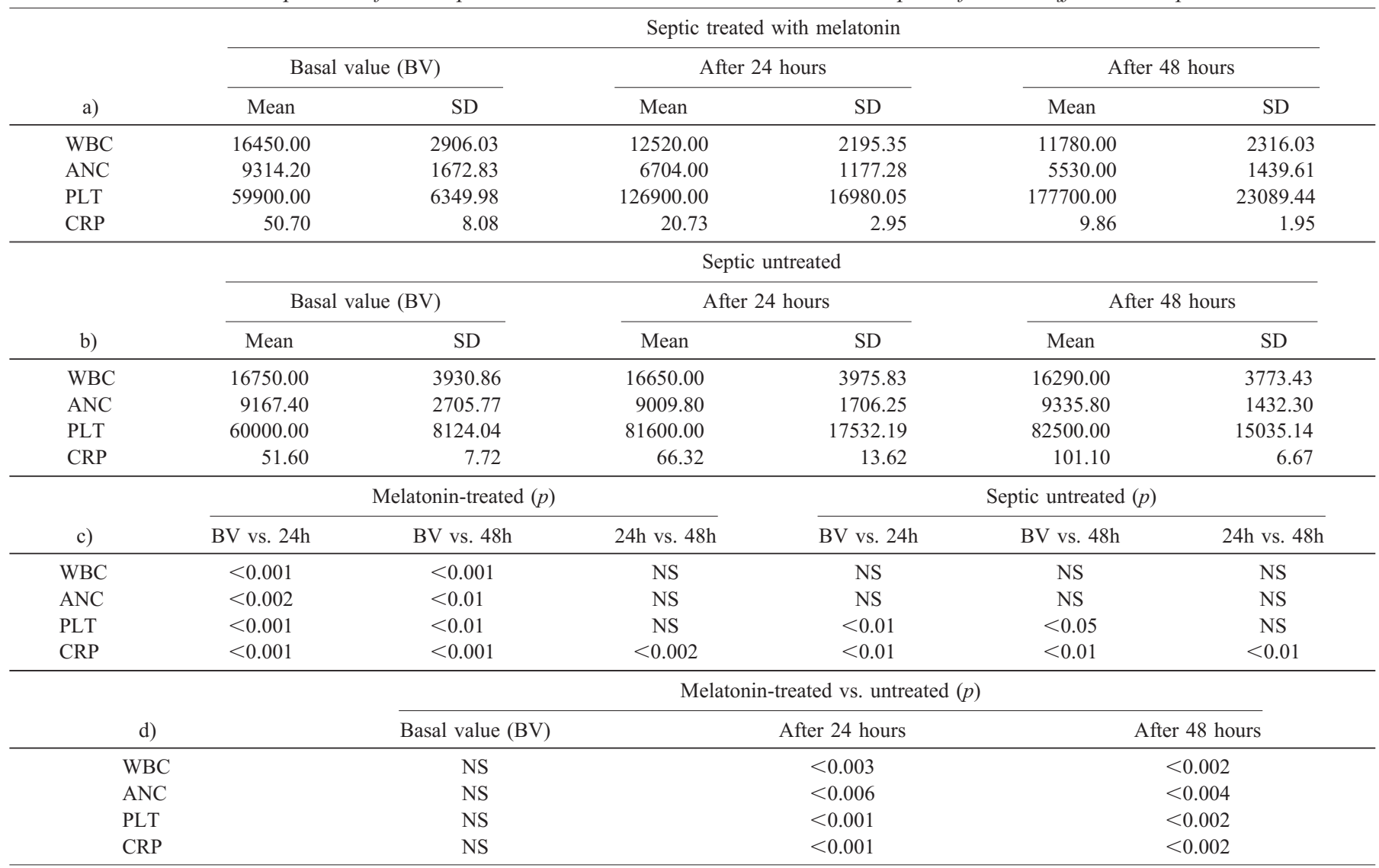

WBC, white blood cell count; ANC, absolute neutrophil count; PLT, platelets; CRP, C reactive protein.

newborns. In the melatonin-treated newborns, the total white blood cell (WBC) count, the absolute neutrophil count (ANC) and the $\mathrm{C}$ reactive protein (CRP) were significantly reduced while platelets increased $24 \mathrm{~h}$ after melatonin administration relative to basal values. This improvement was maintained and statistically significant after $48 \mathrm{~h}$. Conversely, in the untreated septic patients the WBC and ANC were not diminished and the CRP doubled within $48 \mathrm{~h}$. Only the platelet counts increased significantly. Table $3 \mathrm{c}$ shows the significance values between the different time points in the treated and melatonin-untreated septic groups, while Table $3 \mathrm{~d}$ compares the differences between melatonin-treated and untreated newborns at the same time points; these data document the significant $(p<0.01)$ changes that occurred after the administration of melatonin (both at 24 and $48 \mathrm{~h}$ ). It should be noted that no significant differences in serum parameters were present before treatment (basal value).

Table 4 compares the sepsis score defined in Table 1 between septic neonates who were given melatonin and those who were not treated. The initial conditions were virtually identical with 7 treated and 6 untreated patients in the highprobability sepsis (HPS) group and with 3 treated and 4 untreated patients in the probable sepsis (PRS) group, respectively. Three out of 10 patients in both the treated and untreated septic patient groups (total of 6 of 20) had a positive blood culture. It is apparent that, while all 10 untreated patients remained in the PRS group at 24 and $48 \mathrm{~h}$, only 3 of the treated infants remained in the PRS group $24 \mathrm{~h}$ after melatonin administration while 7 were upgraded to the possible sepsis (POS) group. After $48 \mathrm{~h}, 6$ of the melatonin-treated patients were apparently without sepsis (nonsepsis or NS group) while 4 remained in the POS group.

Figure 1 summarizes the serum levels of MDA + 4-HDA in healthy controls, untreated septic newborns and melatonintreated septic newborns. All septic newborns (both the untreated as well as those that were given melatonin subsequently) had elevated serum levels of MDA + 4-HDA ( $p<$ $0.05)$ when compared with healthy controls. These lipid per-

Table 4. Evolution of sepsis score of treated and untreated septic infants at different time points

\begin{tabular}{|c|c|c|c|c|c|c|}
\hline & \multicolumn{2}{|c|}{ Initial conditions } & \multicolumn{2}{|c|}{ After 24 hours } & \multicolumn{2}{|c|}{ After 48 hours } \\
\hline & Melatonin & Untreated & Melatonin & Untreated & Melatonin & Untreated \\
\hline HPS & 7 & 6 & & & & \\
\hline POS & & & 7 & & 4 & \\
\hline NS & & & & & 6 & \\
\hline
\end{tabular}




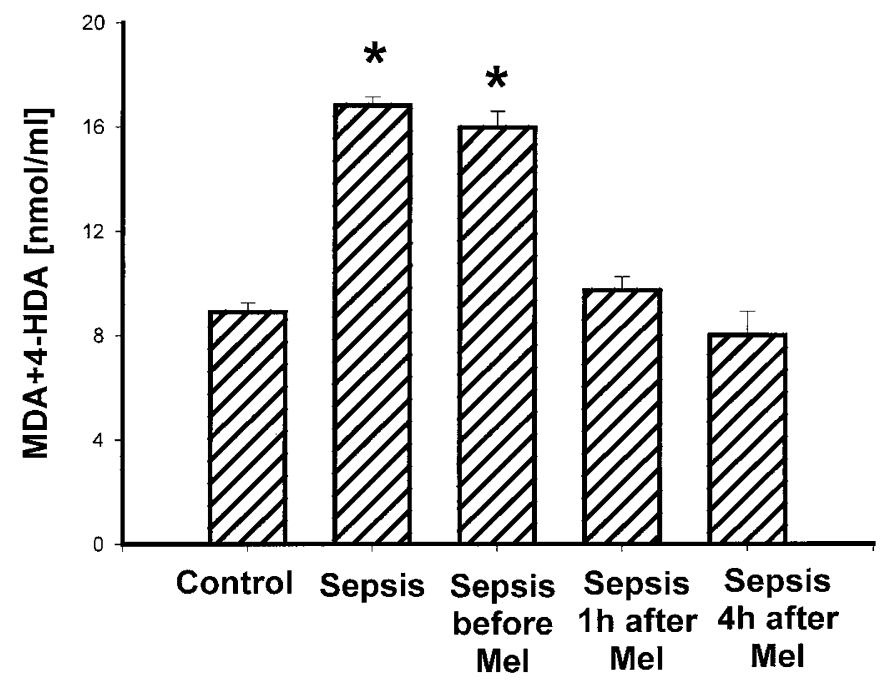

Figure 1. Mean $( \pm \mathrm{SD})$ levels of lipid peroxidation products (MDA + 4-HDA) in the serum of healthy newborns (Control), untreated septic newborns (Sepsis), treated septic infants before melatonin administration (Sepsis before Mel), and finally $1 \mathrm{~h}$ (Sepsis $1 \mathrm{~h}$ after Mel) and $4 \mathrm{~h}$ after melatonin treatment (Sepsis $4 \mathrm{~h}$ after Mel). The two columns identified with an asterisk (*) differ significantly $(p<0.05)$ from groups not marked with an asterisk.

oxidation products were roughly 2-fold higher than those measured in blood of the healthy infants. After treatment with melatonin there was a significant reduction in the levels of lipid peroxidation products at both 1 and $4 \mathrm{~h}$ after treatment $(p<$ $0.05)$.

At $72 \mathrm{~h}$ after the diagnosis of sepsis, 3 of 10 non-melatonin treated children had died, while all the melatonin-treated newborns survived, and continue to do so.

\section{DISCUSSION}

Sepsis is a relatively frequent event in newborns admitted to neonatal intensive care units and is associated with a high rate of morbidity and mortality (1). When septic infection occurs, microorganisms invade the blood stream and release various substances that in turn activate the endogenous mediators of the host systemic response. Sepsis may present with only mild systemic symptoms or progress to septic shock and/or multiple organ failure, which are associated with a mortality rate of $50 \%$ or greater.

Many reports suggest that ROS play an important role in the pathogenesis of sepsis and its complications. Experimental and clinical studies have shown that any harmful tissue event (infection, trauma, anoxia) is perceived by macrophages and monocytes, which in turn secrete cytokines such as interlenkin-1 (IL-1) and tumor necrosis factor (TNF)-alpha. IL-1 and TNF-alpha activate inflammatory cells (neutrophils, macrophages/monocytes, platelets, mastocytes) that release large amounts of toxic oxidizing substances. These reactive oxygen species cause cellular injury via several mechanisms including peroxidation of membrane lipids as well as oxidative damage of proteins and DNA. Batra et al. (4) have documented increased production of ROS in septic neonates. Similarly, Seema et al. (3) found in newborns with sepsis significantly higher levels of TNF-alpha and increased activity of antioxi- dative enzymes, superoxide dismutase, and glutathione peroxidase.

Melatonin, as a free radical scavenger, is believed to work via electron donation to directly detoxify ROS such as the highly toxic hydroxyl radical (7-11) as well as its precursor hydrogen peroxide (12). While melatonin scavenges the hydroxyl radical both in vitro (11) as well as in vivo $(7,13)$, whether this action of melatonin was important in protecting against sepsis in the current study is not known. Melatonin also detoxifies a number of other oxidizing agents including the peroxynitrite anion, singlet oxygen, nitric oxide, and peroxynitrous acid $(8-10,14)$. Besides these scavenging actions, melatonin also stimulates several antioxidative enzymes including superoxide dismutase, glutathione peroxidase, glutathione reductase $(8,10,15)$, and $\gamma$-glutamylcysteine synthase (16), the rate-limiting enzyme in glutathione synthesis. Again, how or whether these multiple antioxidative actions of melatonin contributed to its protective actions in this study remain unknown. The other fact that should be noted is that the septic infants treated with melatonin also received a 1:90 mixture of ethanol/physiologic saline (twice), which was not received by the septic infants not treated with melatonin. The total amount of ethanol given was $110 \mu \mathrm{L}(2 \times 55 \mu \mathrm{L})$. While ethanol itself is reported to have antioxidative actions, considering the very small amount of ethanol these infants received, i.e. $110 \mu \mathrm{L}$, it is unlikely it had a significant effect in terms of antioxidant protection.

In the current study we tested for the first time whether melatonin would modify serum inflammation parameters and improve the clinical course of septic infants as judged by a composite score (Table 1). These data are summarized in Tables 3 and 4 .

The comparison of serum parameters between melatonintreated and untreated septic newborns indeed confirms the anti-inflammatory effect of melatonin (Tables $3 a$ and $3 b$ ). WBC and the ANC significantly decreased in the melatonintreated infants, but they remained elevated in the untreated infants (Table 3c). The CRP level actually doubled after $48 \mathrm{~h}$ in untreated septic infants, while it dropped to $20 \%$ of its basal value in melatonin-treated infants (Table 3c). Finally, data in Table $3 \mathrm{~d}$ confirm that the serum parameters were similar before melatonin administration in the 20 septic newborns enrolled in this study and also show that a significant improvement in these parameters was already seen after $24 \mathrm{~h}$ in those who received melatonin.

Table 4 illustrates the clinical course of sepsis in melatonintreated and untreated newborns during the $48 \mathrm{~h}$ after melatonin administration. Initially, the 20 septic infants were all in the HPS or PRS sepsis state and there were no significant differences between the groups. The data indicate that the 10 infants treated with melatonin improved quickly such that at $24 \mathrm{~h}$ after treatment onset, 7 of them with HPS were upgraded to POS. Additionally, after $48 \mathrm{~h}$ only 4 of the 10 infants remained in the POS state, while 6 exhibited no signs of sepsis (NS). No similar improvement was observed in the 10 septic newborns that did not receive melatonin. These infants all remained in the PRS state at both 24 and $48 \mathrm{~h}$. 
Free radical and reactive species generated during sepsis induce lipid peroxidation of cell membranes which leads to increased serum levels of MDA and 4-HDA as reported previously $(3,4)$ and as seen in the current study. Figure 1 shows that serum MDA + 4-HDA levels were 2-fold higher in septic newborns before melatonin treatment, when compared with the healthy controls. At both 1 and $4 \mathrm{~h}$ following melatonin administration, the levels of lipid peroxidation products, MDA + 4-HDA, had returned to those in normal controls. These changes are consistent with melatonin's ability to reduce the peroxidative breakdown of lipids in cell membranes $(8-10)$. Also, in animal models of sepsis, melatonin is remarkably protective. In rats treated with lipopolysaccharide $(17,18)$ or carrageenan (19), both of which cause massive tissue damage and multiple organ failure, melatonin was found to prevent oxidative damage and to maintain tissue and organ function. Okatani et al. (20) has suggested the treatment of women with preeclampsia with melatonin since in these individuals, as with septic patients, oxidative toxicity and serum levels of peroxidized lipids are elevated.

Of importance to these findings is information related to the potential toxicity of melatonin. A variety of studies including those in children and adult humans (21-23) have shown that melatonin has low toxicity. In a recent study conducted under the guidelines of the U.S. National Toxicology Program, Jahnke et al. (24) found little evidence of toxicity in rats treated throughout pregnancy with massive doses (10 to $200 \mathrm{mg} / \mathrm{kg}$ daily) of melatonin. In addition to maternal health, this group examined prenatal survival, fetal body weight, and incidence of fetal malformations; none of these indices indicated melatonin had any significant toxicity. Considering the findings reported here along with the apparent low toxicity of melatonin, additional trials with this indolamine in larger numbers of septic newborns should be conducted to further test its efficacy as a treatment in conditions where oxidative stress may be elevated.

\section{REFERENCES}

1. Perez EM, Weisman LE 1997 Novel approaches to the prevention and therapy of neonatal bacterial sepsis. Clin Perinatol 24:213-225

2. Antonielli M 1999 Sepsis and septic shock: pro-inflammatory or anti-inflammatory state? J Chemother 6:536-540

3. Seema KR, Mandal RN, Tandon A, Randhawa VS, Mehta G, Batra S, Ray GN, Kapor AK 1999 Serum TNF-alpha and free radical scavengers in neonatal septicemia. Indian J Pediatr 66:511-516
4. Batra S, Kumar R, Seema, Kapoor AK, Ray G 2000 Alterations in antioxidant status during neonatal sepsis. Ann Trop Paediatr 20:27-33

5. Bohles H 1997 Antioxidative vitamins in prematurely and maturely born infants. Int J Vit Nutr Res 67:321-328

6. Hara K, Yamashita S, Fujisawa A, Ishiwa S, Ogawa T, Yamamoto Y 1999 Oxidative stress in newborn infants with and without asphyxia as measured by plasma antioxidants and free fatty acids. Biochem Biophys Res Commun 257:244-248

7. Tan DX, Manchester LC, Reiter RJ, Plummer BF, Hardies LJ, Weintraub ST, Vijayalaxmi, Shepherd AMM 1998 A novel melatonin metabolite, cyclic 3-hydroxymelatonin: A biomarker of melatonin interaction with hydroxyl radicals. Biochem Biophys Res Commun 253:614-620

8. Reiter RJ, Tang L, Garcia JJ, Munoz-Hoyos A 1997 Pharmacological actions of melatonin in free radical pathophysiology. Life Sci 60:2255-2271

9. Reiter RJ, Tan DX, Osuna C, Gitto E 2000 Actions of melatonin in the reduction of oxidative stress: a review. J Biomed Sci 7:444-458

10. Reiter RJ 2000 Melatonin: lowering the high price of free radicals News Physiol Sci $15: 246-250$

11. Tan DX, Chen LD, Poeggeler B, Manchester LC, Reiter RJ 1993 Melatonin: a potent, endogenous hydroxyl radical scavenger. Endocrine J 1:57-60

12. Tan DX, Manchester LC, Reiter RJ, Plummer BF, Limson J, Weintraub S, Qi W 2000 Melatonin directly scavenges hydrogen peroxide: A potentially new metabolic pathway of melatonin biotransformation. Free Radic Biol Med 29:1177-1185

13. Bandyopadhyay D, Biswas K, Bandyopadhyay U, Reiter RJ, Banerjee RK 2000 Melatonin protects against stress-induced lesions by scavenging the hydroxyl radical. J Pineal Res 29:143-159

14. Cuzzocrea S, Costantino G, Caputi AP 1998 Protective effect of melatonin on cellular energy depletion mediated by peroxynitrite and poly (ADP-ribose) synthetase activation in a non-septic shock model induced by zymosan in the rat. J Pineal Res 25:78-85

15. Tan DX, Manchester LC, Reiter RJ, Qi W, Karbownik M, Calvo JR 2000 Significance of melatonin in antioxidative defense system: Reactions and products. Biol Signals Recept 9:137-159

16. Urata Y, Homma S, Goto S, Todoroki S, Vedu T, Cho T, Honma K, Kondo T 1999 Melatonin induces $\gamma$-glutamylcysteine synthetase mediated by activator protein- 1 in human vascular endothelial cells. Free Radic Biol Med 27:838-847

17. Sewerynek E, Abe M, Reiter RJ, Barlow-Walden LR, Chen LD, McCabe TJ, Roman LJ, Diaz-Lopez B 1995 Melatonin administration prevents lipopolysaccharideinduced oxidative damage in phenobarbital-treated rats. J Cell Biochem 58:436-444

18. Sewerynek E, Melchiorri D, Chen LD, Reiter RJ 1995 Melatonin reduces both basal and bacterial lipopolysaccharide-induced lipid peroxidation. Free Radic Biol Med 19:903-909

19. Cuzzocrea S, Tan DX, Costantino G, Mazzon E, Caputi AP, Reiter RJ 1999 The protective role of endogenous melatonin in carrageenan-induced pleurisy in the rat. FASEB J 13:1930-1938

20. Okatani Y, Watanabe K, Hayashi K, Wakatsuki A, Sagara Y 1997 Melatonin suppresses vasospastic effect of hydrogen peroxide in human umbilical artery: relation to calcium influx. J Pineal Res 22:232-237

21. Molina-Carballo A, Muñoz-Hoyos A, Reiter RJ, Sanchez-Forte M, Moreno-Madrid F, Rufo-Campos M, Molina-Font JA, Acuña-Castroviejo D 1997 Utility of high doses of melatonin as adjunctive anticonvulsant therapy in a child with severe myoclonic epilepsy: two years' experience. J Pineal Res 23:97-105

22. Jan JE, Hamilton D, Seward N, Fast DK, Freeman RD, Laudon M 2000 Clinical trials of controlled-release melatonin in children with sleep-wake cycle disorders. J Pineal Res 29:34-39

23. de Lourdes M, Seabra V, Bignotto M, Pinto LR Jr, Tufik S 2000 Randomized double-blind clinical trial, controlled with placebo, of the toxicology of chronic melatonin treatment. J Pineal Res 29:193-200

24. Jahnke G, Marr M, Myers C, Wilson R, Travlos G, Price C 1999 Maternal and developmental toxicity evaluation of melatonin administered orally to pregnant Sprague-Dawley rats. Toxicol Res 50:271-274 